\title{
artigo
}

Guimarães, G.L.P.; Pereira, R.S.; Paula, E.J.C.; Mendes, G.N.; Peixoto, M.B.; Spagnol, C.A.;

A importância da gestão documental em unidades de terapia intensiva

\section{A importância da gestão documental em unidades de terapia intensiva}

\author{
Documental management and its importance in intensive care units
}

La gestión documental y su importancia en las unidades de cuidados intensivos

\begin{abstract}
RESUMO
Objetivos: descrever como ocorre a gestão documental na Unidade Terapia Intensiva e identificar fatores facilitadores e dificultadores da gestão documental no cotidiano de trabalho. Método: Revisão narrativa da literatura realizada no período de julho a setembro de 2020, nas bases de dados do Ministério da Saúde, Google acadêmico, SciELO e Medline. Foram encontrados 198 artigos e selecionados 04. Os dados foram organizados e analisados à luz da literatura científica. Resultados: Destaca-se a diversidade de periódicos que os artigos analisados foram publicados: Revista Acervo; Revista Saúde digital e tecnologia educacional; Texto e Contexto em Enfermagem e Revista de Iniciação Científica. Conclusão: a gestão documental se mostrou capaz de aprimorar o arquivamento e utilização de documentos de forma qualificada, diminuindo o tempo e a mão de obra empregada na busca de documentos perdidos.
\end{abstract}

DESCRITORES: Gerenciamento de dados; Unidades de terapia intensiva; Prontuários; Registros Eletrônicos de Saúde.

\section{ABSTRACT}

Objectives: To describe how document management occurs in the Intensive Care Unit and to identify factors that facilitate and hinder document management in daily work. Method: Narrative review of the literature carried out from July to September 2020, in the databases of the Ministry of Health, Google Scholar, SciELO and Medline. 198 articles were found and selected 04. The data were organized and analyzed in the light of the scientific literature. Results: The diversity of journals that the analyzed articles were published stands out: Revista Acervo; Digital Health and Educational Technology Magazine; Text and Context in Nursing and Scientific Initiation Magazine.Conclusion: The present study provided an insight into the documentary and archival processes in the intensive care unit and the importance of document management considering its definition as well as its stages and aspects.

DESCRIPTORS: Data management; Intensive care unit; Medical Records; Electronic Health Records.

\section{RESUMEN}

Objetivos: Comprender cómo se produce la gestión documental en la Unidad de Cuidados Intensivos e identificar los factores que facilitan y dificultan la gestión documental no diaria del trabajo. Método: Revisión de literatura narrativa realizada de julio a septiembre de 2020, con base en las bases de datos del Ministerio de Salud, Google Académico, SciELO y Medline. Se encontraron 198 artículos y seleccionaron 04. Los datos se organizan y analizan a la luz de la literatura científica. Resultados: Destacamos la diversidad de periódicos en los que se publicaron los artículos analizados: Revista Acervo; Revista digital Tecnología sanitaria y educativa; Revista Texto y contexto en enfermería e iniciación científica. Conclusión: El presente estudio permitió conocer los procesos documentales y de archivo en la unidad de cuidados intensivos y la importancia de la Gestión Documental considerando su definición asi como sus etapas y aspectos.

DESCRIPTORES: Manejo de Datos; unidad de cuidados intensivos; história clínica; Registros Electrónicos de Salud.

RECEBIDO EM: 08/10/2020 APROVADO EM: 09/11/2020

\section{Gabriel Lucas Pereira Guimarães}

Tecnólogo em Secretariado, Pós-graduando em Administração e Auditoria em Serviços de Saúde, Graduando em Enfermagem pela Faculdade Adventista da Bahia. BA-Brasil.

ORCID: 0000-0002-4669-6715 


\section{Raquel Silva Pereira}

Graduanda de Enfermagem pela Faculdade Adventista da Bahia. BA-Brasil.

ORCID: 0000-0002-9530-1855

\section{Emilly Jhully Correia de Paula}

Graduanda de Enfermagem pela Faculdade Adventista da Bahia. BA-Brasil.

ORCID: 0000-0002-6599-9412

\section{Giovanna Nascimento Mendes}

Graduanda de Odontologia pela Faculdade Adventista da Bahia. BA-Brasil.

ORCID: 0000-0002-6872-6129

\section{Marcela Barbosa Peixoto}

Enfermeira. Especialista em Enfermagem Intensiva-UTI. Coordenadora de Enfermagem e RT da UTI do Hospital e Maternidade Luiz Argolo - SAJ/BA. Docente do curso de Enfermagem da Faculdade Adventista da Bahia. BA-Brasil.

ORCID: 0000-0002-8807-0833

\section{Carla Aparecida Spagnol}

Enfermeira. Professora Associada da Escola de Enfermagem da Universidade Federal de Minas Gerais. Doutora em Saúde Coletiva pela Universidade Estadual de Campinas com período sanduíche na Université Vincennes Saint Denis Paris 8. Pós-doutorado na Université Cergy Paris. Minas Gerais-Brasil.

ORCID: 0000-0003-1588-2109

\section{INTRODUÇÃO}

$\mathbf{N}$ as organizações $90 \%$ das suas informações estão contidas em documentos e como $70 \%$ destas não são capazes de resistir a uma catástrofe, como incêndio ou enchentes, na maioria das vezes, há uma perda em massa da sua documentação. Sendo assim, para manter a ordem e garantir a integridade de tais documentos, torna-se fundamental a implementação de um sistema de gestão documental (GD) efetivo nas empresas públicas ou privadas ${ }^{(1,2)}$.

Segundo a Lei no 8.159 , de 8 de janeiro de 1991, a GD é definida como um "conjunto de procedimentos e operações técnicas à sua produção, tramitação, uso, avaliação e arquivamento em fase corrente e intermediária, visando a sua eliminação ou recolhimento para guarda permanente" (3). São medidas rotineiras que buscam racionalizar e potencializar a eficiência na produção, classificação, análise, preservação, acesso e uso das informações registradas em documentos de arquivo, a qual perpassa por três etapas, sendo elas: produção, utilização e destinação dos documentos ${ }^{(4)}$.

$\mathrm{Na}$ área da saúde, embora pouco se fale a respeito da GD, o Departamento de Trabalho dos Estados Unidos apontou que há uma perda ou uma má utilização de documentos imprescindíveis à continuidade da assistên- cia nos serviços de saúde, o que desencadeia uma série de custos e erros no processo de trabalho. Por exemplo, a cada 20 documentos, um pode ser perdido e para recuperá-lo gasta-se cerca de 25 horas. Além disso, de 10 a $12 \%$ dos documentos não são encontrados na primeira tentativa, ocupando um tempo médio de 400 horas por funcionário a cada ano. Ressalta-se que estas horas dispendidas além de gerarem gastos desnecessários para a empresa poderiam ser melhor utilizadas na prestação de serviços ${ }^{(2)}$.

Assim, com o objetivo de melhorar os processos de trabalho, a partir da utilização das tecnologias de informação em saúde, no Brasil foram estabelecidas as diretrizes para a GD e o sistemas de arquivos, na Política Nacional de Informação e Informática em Saúde (PNIIS) e no $2^{\circ}$ Plano Diretor para o Desenvolvimento da Informação e Tecnologia de Informação em Saúde (2 ${ }^{\circ} \mathrm{Pla}-$ DITIS 2013 - 2017), definindo assim seus objetivos, finalidades e responsabilidades que deveriam ser seguidas por entidades públicas e privadas no âmbito do Sistema Único de Saúde (SUS), com o intuito de aprimorar a governança das informações e dos recursos de informática. ${ }^{(5,6)}$.

A Unidade de Terapia Intensiva (UTI), consiste em um setor que reúne informações complexas e importantes, principalmen- te, nos prontuários dos pacientes, os quais fornecem subsídios técnico e legal para a assistência. $\mathrm{O}$ prontuário com informações fidedignas constitui-se em ferramentas que possibilitam a equipe de saúde obter orientação e direcionamento para as suas condutas terapêuticas em alta complexidade, logo vê-se a importância da evolução das tecnologias da informação aplicadas a manutenção da legibilidade, e bom uso destes documentos. A lei n ${ }^{\circ} 13.787$, de 27 de dezembro de 2018, institui que tais documentos devem ser mantidos no período de 20 anos após a última anotação realizada, sendo obrigatório durante este período, ainda que o paciente venha a óbito, portanto, é mais que imprescindível desempenhar um processo de armazenamento detalhado e organizado, como o proposto pela $\mathrm{GD}^{(7,8)}$.

Os objetivos elaborados para este estudo foram: descrever como ocorre a gestão documental na UTI e identificar fatores facilitadores e dificultadores da GD no cotidiano de trabalho.

\section{MÉTODO}

O presente estudo é uma revisão narrativa da literatura realizada no período de julho a setembro de 2020, a partir de um levantamento nas bases de dados do Ministério da Saúde, Google acadêmico, SciELO e Medline. 


\section{artigo}

Guimarães, G.L.P.; Pereira, R.S.; Paula, E.J.C.; Mendes, G.N.; Peixoto, M.B.; Spagnol, C.A.;

A importância da gestão documental em unidades de terapia intensiva

Este tipo de revisão "apresenta uma temática mais aberta; dificilmente parte de uma questão específica bem definida, não exigindo um protocolo rígido para sua confecção; a busca das fontes não é pré-determinada e específica (...)" (9). Tem a vantagem de permitir ao pesquisador uma apropriação de seu objeto de estudo, a partir de uma busca abrangente sobre o fenômeno a ser estudado ${ }^{(10)}$.

Foram adotados como critérios de inclusão: artigos na íntegra e indexados que tratavam da temática proposta, publicados no período de 2010 a 2020, em português. E como critério de exclusão: artigos duplicados, teses, monografias e resumos de anais.

A partir do material encontrado foram lidos os títulos de 198 artigos e seleciona- dos aqueles que atendiam o tema. Após realizou-se a leitura dos resumos, sendo selecionados 08 que atenderam aos critérios de inclusão. Em seguida foi feita uma leitura completa e cuidadosa de 07 artigos, mas somente 04 foram selecionados, utilizando um instrumento para sintetizar as informações necessárias ao estudo.

Os dados encontrados foram organizados e analisados de forma crítica, a partir da interpretação do compilado dos resultados comparando-os com a literatura científica. A apresentação dos resultados foi através de descrição para possível comparação entre todos os estudos selecionados. Segue abaixo o fluxograma do percurso metodológico (Figura 01).
Figura 1. Fluxograma das informações da pesquisa nas bases, mapeando o número de registros identificados, incluídos e excluídos, e os motivos para exclusões, de acordo com o PRISMA Flow Diagram. Cachoeira, BA, Brasil, 2020.

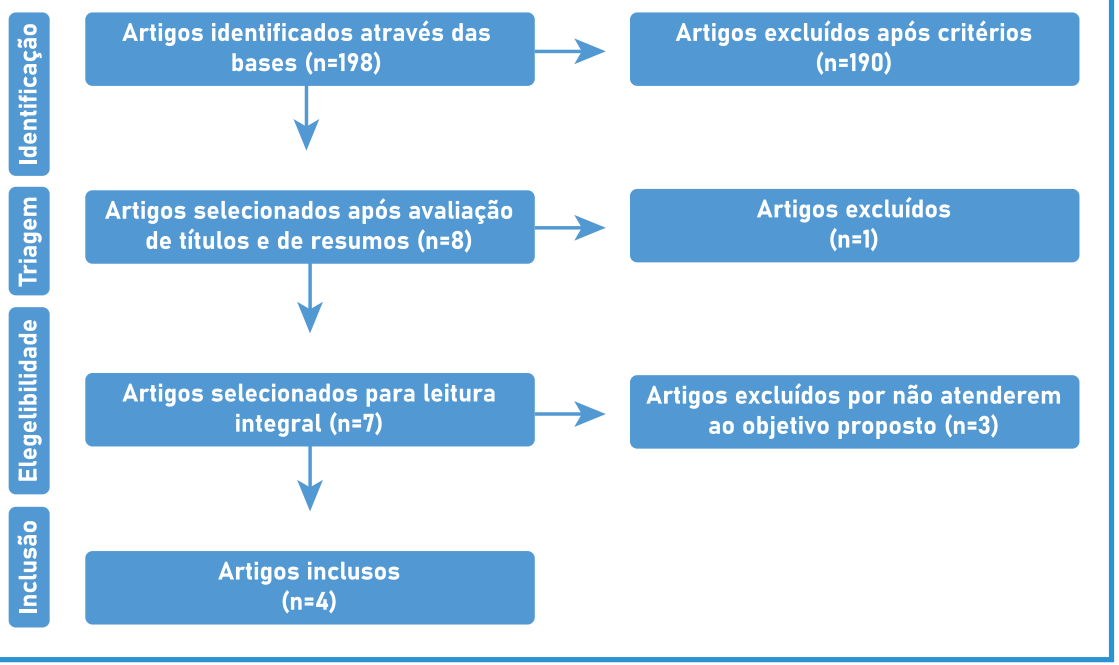

\section{RESULTADOS}

A figura 2 apresenta os 04 artigos selecionados, com os seus respectivos autores, títulos, local do estudo, ano e periódicos em que foram publicados.

Verifica-se que 03 estudos foram realizados em Santa Catarina e 01 no estado da Bahia. O ano de publicação variou de 2009 a 2018.

Destaca-se a diversidade de periódicos que os artigos analisados foram publicados: Revista Acervo; Revista Saúde digital e tecnologia educacional; Texto e Contexto em Enfermagem e Revista de Iniciação Científica.

\section{DISCUSSÃO}

\section{As etapas da GD no contexto das uni- dades de terapia intensiva}

De modo geral a GD se divide em três etapas que se articulam para promover o funcionamento adequado e a otimização dos serviços ${ }^{(4)}$, a fim de auxiliar as funções dos setores administrativo e legais/fiscais, dado o seu valor histórico respectivamente após a sua avaliação ${ }^{(11,12)}$.

A etapa inicial, intitulada produção de documentos, compreende a criação, preparação e gestão dos registros, e possibilita a prevenção da produção de documentos irrelevantes, aqui também se dá a implementação de tecnologias no processo de criação documental. Na área da saúde, é nessa etapa que os profissionais de saúde das mais variadas áreas de atuação recepcionam o paciente e fazem seus direcionamentos e prescrições em prontuário sendo este um documento de manutenção permanente ${ }^{(7,4,12)}$.

Quadro 1. Resultados encontrados nos estudos de acordo com o autor, o título, o ano de publicação, o local do estudo e o periódico em que foi publicado. Cachoeira (BA), Brasil, 2020.

\begin{tabular}{|c|c|c|c|c|}
\hline CÓDIGO & AUTOR & TíTULO & LOCAL & PERIÓDICO/ANO \\
\hline 01 & $\begin{array}{c}\text { Cunha FJAP, Oliveira LAF, } \\
\text { Lima GLQ }\end{array}$ & A função de avaliação na gestão documental em hospitais & Bahia & Acervo 2015. \\
\hline 02 & $\begin{array}{c}\text { Oribka R, Cruz ASC, } \\
\text { Bahia, EMS }\end{array}$ & $\begin{array}{c}\text { Procedimentos da gestão documental aplicados no } \\
\text { arquivo de prontuários dos pacientes do hospital } \\
\text { universitário da Universidade Federal de Santa Catarina }\end{array}$ & $\begin{array}{c}\text { Santa Catarina } \\
\text { Re. saúd. digi. tec. } \\
\text { edu. } 2018 .\end{array}$ \\
\hline 03 & $\begin{array}{c}\text { Lorenzetti J, Trindade LL, } \\
\text { Pires DEP, Ramos FRS }\end{array}$ & $\begin{array}{c}\text { Tecnologia, inovação tecnológica e saúde: uma reflexão } \\
\text { necessária }\end{array}$ & Santa Catarina & $\begin{array}{c}\text { Texto e Contexto em } \\
\text { Enfermagem.2012. }\end{array}$ \\
\hline Rocha EL, et al. & $\begin{array}{c}\text { Prontuário eletrônico para acompanhamento médico } \\
\text { dos pacientes de uma unidade de terapia intensiva. }\end{array}$ & Santa Catarina & $\begin{array}{c}\text { Revista de Iniciação } \\
\text { Científica. 2009. }\end{array}$ \\
\hline
\end{tabular}


A segunda etapa corresponde à utilização dos documentos, nesta fase haverá a "criação e melhoramento dos sistemas de arquivos, recuperação de dados, produção e manutenção de programas de documentos vitais" (4). Esta fase busca assegurar a seleção adequada dos materiais e o momento em que os profissionais têm acesso às informações já registradas no prontuário, com o objetivo de dar sequência ao processo do cuidado baseado no histórico do paciente. Nesta etapa também poderá haver modificação e adição de novas informações, onde prima-se por rapidez na disposição destas de forma documentada e com o devido arquivamento ${ }^{(4,12,13)}$.

Por fim, a terceira etapa trata da destinação de documentos que se refere à descrição das séries documentais, na avaliação de documentos, destinação destes ao arquivamento, e eliminação ou recolhimento dos arquivos classificando-os como permanentes. Nesta etapa a unidade adotará métodos para dar destino aos conteúdos do prontuário, fazendo revisões, eliminações ou direcionando os documentos aos arquivos permanentes da $\mathrm{UTI}^{(4,11)}$

Compreender como se dá a GD na UTI possibilita melhor entendimento sobre as dificuldades do setor bem como as oportunidades de melhoramento ao mesmo tempo viabiliza uma gestão eficaz da unidade.

\section{Fatores facilitadores e dificultadores da GD no cotidiano de trabalho da UTI}

A GD é um fator de grande influência na vitalidade das organizações de saúde, pois os arquivos das instituições não possuem finalidade meramente administrativas, possuindo também uma grande importância no contexto histórico, bases para aprendizado, inovações organizacionais e desenvolvimento científico ${ }^{(4,12)}$. É de extrema importância salientar que a GD em sua etapa de avaliação destina documentos de acordo com o seu objetivo primário ou secundário, e proporciona grandes melhorias, propiciando a seleção de documentos que são realmente indispensáveis, eliminando assim os que não possuem utilidade, fazendo com que haja uma diminuição no acúmulo de documentos, favorecendo a funcionalidade e operacionalização dos mesmos ${ }^{(11)}$.

Os inúmeros avanços e investimentos na área técnico-científica no âmbito da saúde tem se tornado cada vez mais crescentes, a informação e comunicação instantânea, prontuário eletrônico único nacional e integrado para acesso internacional, são algumas das principais contribuições. Diversos estudos envolvendo as UTI's como campo específico, têm evidenciado os constantes desafios e situações complexas vivenciadas dentro deste ambiente que exigem uma postura de maior segurança e controle dos profissionais de saúde. É neste contexto que a integração correta das inovações tecnológicas tem contribuído para uma melhor assistência ao paciente com uma maior facilidade e desenvoltura na organização da equipe que irá assistir ao mesmo ${ }^{(14)}$.

Dentre as principais inovações tecnológicas integradas a assistência em saúde, podemos citar o Gerenciamento Eletrônico de Documentos (GED), sendo este capaz de auxiliar na segunda etapa de desenvolvimento do GD. O GED possibilita não somente guardar os documentos que já foram criados de forma digital, mas também contribui para solucionar o problema criado pela produção excessiva de papel (documentos comprobatórios), nas instituições públicas e privadas. Além disso, as melhorias do GED constituem-se em um grande divisor de águas dentre as equipes no ambiente hospitalar, dentre estas, citamos o Prontuário Eletrônico do Paciente (PEP), que tem se tornado um grande aliado na assistência aos usuários que integram as alas das UTIs ${ }^{(15)}$.

A implementação do PEP oferece inúmeros benefícios aos seus usuários, como segurança, qualidade, otimização de custos, além de facilitar o armazenamento permanente e a recuperação de informações, garantindo a segurança destes, possibilitando também o compartilhamento entre componentes da mesma equipe e/ou outras instituições de maneira rápida, organizada, individualizada e eficaz ${ }^{(13,16)}$.

O processo de tratamento nesse setor é demasiadamente complexo, exigindo o uso de dados em diversas situações. A manipulação de prontuários acentua a possibilidade de erros, e a perda de dados importantes, além de impossibilitar que mais de um profissional consiga acessar e atualizar o quadro clínico de um paciente de forma simultânea ${ }^{(17)}$. O prontuário por ser um documento único compar- tilhado por toda a equipe de saúde, sendo um importante método para a qualificação dos processos de trabalho, contribuindo para a eficácia dos cuidados ao paciente crítico $(12,18)$. A GD nesse contexto é uma importante ferramenta para a prestação dos atendimentos de saúde, contribuindo para o gerenciamento das unidades hospitalares ${ }^{(11,19)}$.

Salienta-se que para o funcionamento das UTI's é necessário que a assistência prestada pelos profissionais da unidade seja registrada, assinada e datada no prontuário do paciente, de forma legível e contendo o carimbo do profissional, evidenciando a importância dos processos arquivísticos da GD, cabendo a unidade em caso de transferência intra-hospitalar disponibilizar os dados do prontuário ao setor de destino ${ }^{(4,12,20)}$.

Portanto, os prontuários eletrônicos possibilitam alcançar um maior nível de qualidade frente aos cuidados de saúde, afinal, alguns destes interagem com o prescritor, indicando interações medicamentosas inadequadas, bloqueando vias incorretas de administração, além de facilitarem a comunicação multidisciplinar com clareza de dados referentes ao paciente, padronizando processos médicos e possibilitando suporte para diagnósticos, permitindo prevenção de erros, ao contrário do prontuário convencional, principalmente no âmbito das UTIs ${ }^{(21)}$.

Nesta perspectiva, a GD quando bem implantada e utilizada adequadamente beneficia o desenvolvimento administrativo, agregando eficiência nas auditorias, sejam elas externas ou internas, afinal, uma das principais ferramentas utilizadas nestas auditorias são os prontuários ${ }^{(22,23)}$.

O foco central da auditoria no ambiente hospitalar, seja ela interna ou externa, é contribuir em todas as áreas para a gestão da organização, trabalhando assim para melhorias na assistência, na eficiência e na oferta de serviços de saúde. A auditoria sempre faz uma avaliação ampla, sistemática e formal das atividades dos profissionais, objetivando a valorização do sistema de educação e melhoramento contínuo oferecendo qualidade e segurança, onde a GD tem um papel insubstituível para a realização desses objetivos tendo em vista que sua correta aplicação favorece um melhor processo arquivístico e seu adequado uso ${ }^{(4,2,2,23,24)}$. 


\section{artigo}

Guimarães, G.L.P.; Pereira, R.S.; Paula, E.J.C.; Mendes, G.N.; Peixoto, M.B.; Spagnol, C.A.

A importância da gestão documental em unidades de terapia intensiva

\section{CONCLUSÃO}

O presente estudo proporcionou uma visão sobre os processos documentais $\mathrm{e}$ arquivísticos em UTI e a importância da GD, considerando sua definição bem como suas etapas e aspectos, ressaltando o valor e utilização das informações mantidas em documentos utilizados neste setor, as quais possibilitam amparo tanto para profissionais quanto para usuários. Além disso, fornecem embasamento para evolução dos estudos científicos que possibilitam melhorias na prestação do cuidado.

A GD se mostrou como uma ferramenta capaz de aprimorar o arquivamento e utiliza- ção de documentos de forma qualificada, trazendo praticidade e organização para as

instituições, além de diminuir o tempo e a mão de obra empregada na recriação ou busca de documentos perdidos, destinando assim esses recursos para a prestação de outros serviços, como por exemplo, na assistência ao paciente.

\section{REFERÊNCIAS}

1. Calderon WR, Cornelsen JM, Pavezi N, Lopes MA. O processo de gestão documental e da informação arquivística no ambiente universitário. Ci. Inf. 2004;33(3):97-104.

2. Termale D. The Real Costs of Lost Documents, from Konica Minolta [Internet]. [place unknown]: Industry Analysts, Inc.; 2015 [cited 2020 Oct 7]. Available from: https://industryanalysts. com/111015_konica/.

3. Brasil. lei $n^{\circ} 8.159$, de 8 de janeiro de 1991 [Internet]. Brasilia; 1991 Available from: http://www.planalto.gov.br/ccivil_03/leis/ 18159.htm

4. Ministério da cidadania. Manual de Gestão Documental [Internet]. Rio de Janeiro; 2016 [cited 2020 Sep 14]. Available from: https://www.bn.gov.br/documento/mgd

5. Ministério da Saúde. Portaria N 589, de 20 de maio de 2015. Institui a Política Nacional de Informação e Informática em Saúde (PNIIS). [Internet]. Brasilia; 2015 [cited 2020 Oct 27]. Available from: http://bvsms.saude.gov.br/bvs/saudelegis/gm/2015/ prt0589_20_05_2015.html

6. Abrasco. $2^{\circ}$ Plano diretor para o desenvolvimento da informação e tecnologia de informação em saúde [Internet]. Rio de Janeiro: GTISP / Abrasco; 2013 [cited 2020 Sep 10]. Available from: http:// www.abrasco.org.br/ckfinder/userfiles/files/PlaDITIS\%20201317\%202a\%20Versao\%20Consulta\%20publ\%20(1).pd

7. Thofehrn C, Lima WC. Prontuário Prontuário Eletrônico do Paciente - A Importância da Clareza da Informação. RESI. 2006;5(1).

8. Brasil. lei $n^{0} 13.787$, de 27 de dezembro de 2018 [Internet]. Brasilia; 2018 Available from: http://www.planalto.gov.br/ccivil_03/_ato2015-2018/2018/lei/L13787.htm\#: :text=L13787\&text=LEl $\% 20 \mathrm{~N} \% \mathrm{C} 2 \% \mathrm{BA} \% 2013.787 \% 2 \mathrm{C} \% 20 \mathrm{DE} \% 2027$,manuseio $\% 20$ de\%20prontu\%C3\%A1rio\%20de\%20paciente.

9. Cordeiro AM, Oliveira GM, Rentería JM, Guimarães CA. Revisão sistemática: uma revisão narrativa. Rev. Col. Bras. Cir. [Internet]. 2007 Dec [cited 2020 Nov 05] ; 34( 6 ): 428-431. Available from: http://www.scielo.br/scielo.php?script=sci_arttext\&pid=S0100-69912007000600012\&Ing=en

10. Gil AC. Como elaborar projetos de pesquisa. 5 ed. São Paulo: Atlas; 2010.

11. Cunha FJAP, Oliveira LAF, Lima GLQ. A função de avaliação na gestão documental em hospitais. Acervo. 2015;28(2):206-225

12. Oribka R, Cruz ASC, Bahia EMS. Procedimentos da gestão documental aplicados no arquivo de prontuários dos pacientes do hospital universitário da Universidade Federal de Santa Catarina. Re. saúd. digi. tec. edu. 2018;3(especial):82-99.

13.Rocha EL, Jaraceski JC, Charnovscki R, Filho JRN, Nicoleit ER, Simões PWTA et al. Prontuário eletrônico para acompanhamento médico dos pacientes de uma unidade de terapia intensiva. Rev. de Iniciação Científica. 2009;7(1)

14. Lorenzetti J, Trindade LL, Pires DEP, Ramos FRS. Tecnologia, inovação tecnológica e saúde: uma reflexão necessária. Texto Contexto Enferm, Florianópolis, 2012 Abr-Jun; 21(2): 432

15. Thomaz KP, Santos VM. Metadados para o gerenciamento eletrônico de documentos de caráter arquivístico - GED/A: estudo comparativo de modelos e formulação de uma proposta preliminar. Rev. de Ciência da Informação. 2003;4(4)

16. Silva AB, Guedes ACCM, Síndico SRF, Vieira ETRC, Andrade Filha, I.G.F. Registro eletrônico de saúde em hospital de alta complexidade: um relato sobre o processo de implementação na perspectiva da telessaúde. Ciênc. saúde coletiva. 2019; 24(3): 1133-1142.

17. Bahar M, Inel EY. Use of software in the ICU. Turkish journal of trauma and emergency surgery. 2019:535-544.

18. Ministro da saúde. Portarian895, de 31 de março de 2017 [Internet]. Brasilia; 2017 [cited 2020 Sep 10]. Available from: http://www. saude.gov.br/images/pdf/2017/abril/07/106713-16-82-Minuta-Portaria-PROTOCOLO.pdf

19. Mesquita AMO, Deslandes SF. A Construção dos Prontuários como Expressão da Prática dos Profissionais de Saúde. Saúde Soc. 2010;19(3):664-673.

20.Ministro da saúde. Resolução $n^{\circ}$ 7, De 24 de Fevereiro de 2010 [Internet]. Brasília; 2010 [cited 2020 Sep 4]. Available from: http://bvsms.saude.gov.br/bvs/saudelegis/anvisa/2010/ res0007_24_02_2010.html

21. Colleti JJ, Andrade AB, Carvalho WB. Avaliação do uso de sistemas de prontuário eletrônico nas unidades de terapia intensiva brasileiras. Rev. bras. ter. intensiva. 2018; 30(3): 338-346.

22. Borsato FG, Rossaneis MA, Haddad MCFL, Vannuchi MTO, Vituri DW. Avaliação da qualidade das anotações de enfermagem em um Hospital Universitário. Acta paul. enferm. 2011; 24(4): 527-533.

23. Claudino HG, Gouveia EML, Santos S, Lopes MEL. Auditoria em registros de enfermagem: revisão integrativa de literatura. Rev. enferm. UERJ, 2013.

24. Macena JL, Jordão CP, Filho JLJX. Auditoria interna: uma análise da implantação em uma instituição privada de serviço de saúde (Hospital). Rev. pesquisa em administração. 2017;1(1):69-84. 